\title{
Fragen des Christen: \\ Bilanz einer religiösen Sendereihe des ORF
}

von Josef Moosbrugger

\section{Eine Sendereihe entsteht}

Im Juni 1975 läutete das Telefon. Ernst Niesner, damals Leiter der Abteilung Kirche im Fernsehen, rief an, ob ich nicht Lust hätte, bei einer neuen Sendereihe mitzumachen. Man wüßte zwar noch nicht genau, wie sie ausschauen sollte, aber das Wichtigste wäre auf jeden Fall, daß man ganz auf feedback ausgehen wollte, auf Gespräch mit dem Zuschauer. Die Themen sollten aus der Zuschauerschar selbst kommen, und in der letzten Sendung wollte man sogar schon auf die Briefe der vorhergehenden Sendung eingehen. Aber alles andere würde sich ganz von selbst aus den Reaktionen und Erfahrungen ergeben. Diese Fünfminutensendung war also irgendwie eine Nachahmung von Pfarrer Sommerauers Hitsendung aus dem Bayerischen Fernsehen.

Das Team: Das Team der Sprecher bestand zunächst aus sechs, vier Katholiken und zwei Protestanten. Später wurde diese Zahl auf vier reduziert, im Verhältnis 3:1. Die einzelnen Sprecher wurden mit Bedacht stark typisiert. Ein Ordensmann, ein Hochschulseelsorger, ein Großstadtpfarrer, ein Dorfpfarrer. Damit sollten möglichst viele Gruppen sich angesprochen fühlen. Und noch etwas war ganz neu. Das Team selbst sollte in der Vorbereitung, in der Auswertung möglichst zusammenarbeiten. Ein Wunsch, der obwohl von allen ehrlich und sehnlichst ausgesprochen, wegen der großen Entfernungen und der Terminschwierigkeiten, nur sehr schwer realisierbar war und ist. Es zeigt sich immer mehr, daß diese Teamarbeit notwendig und auch sehr fruchtbar ist. Die Zahl 4 der Sprecher hat sich deshalb als günstig erwiesen, weil dadurch die Sprecher einen sehr großen Publizitätsgrad erhalten. Die Teamarbeit ist etwas sehr Wohltuendes, Unterschiede zwischen Katholiken und Protestanten werden überhaupt nicht bemerkt.

Visualisierung: Eine Sendung, die den Zuschauer ansprechen soll, steht und fällt mit dem Gesicht des Sprechers, das heißt mit der menschlichen Qualität, der Ausstrahlung und der Fähigkeit, über ein Problem verständlich und menschlich zu sprechen. Doch wollte man von Anfang an auch das Thema fernsehgerecht visualisieren. Dabei versuchten wir es im 1. Jahr mit einem „Zeugen”, einer Persönlichkeit, die auf dem Gebiet des besprochenen Themas kompetent reden konnte. Dieser Versuch mußte deshalb fallen gelassen werden, weil es sehr schwierig war, geeignete Zeugen zu finden die sich auszudrücken verstanden. So versuchten wir, einen wichtigen Aspekt des Themas auch im Bild zu präsentieren! Eine allseits befriedigende Lösung haben wir bis jetzt noch nicht gefunden. In Michael Weinmann haben wir einen Allrounder der Regie gefunden. Er scheut weder Zeit noch Mühe noch Einfälle, das Wortmanuskript auch in ein Bildmanuskrpt umzuwandeln! Jede Sendung durchläuft in der Vorbereitung einen Dreierschritt. Zunächst werden die möglichen Themen im Team besprochen und

Josef Moosbrugger, 57, ist Pfarrer in einer Dorfgemeinde in Tirol mit 1.200 Einwohnern. Daneben übter eine Lehrtätigkeit für Medienpädagogik und Mediendidaktik an der Theologischen Fakultät Innsbruck und mehreren Höheren Lehranstalten aus. Seit 1970 ist er Sprecher in der Sendung „Christ in der Zeit”, seit 1975 in „Fragen des Christen”. 
ausgewählt, Dann erfolgt, wieder im Team, eine sehr eingehende und offene Diskussion über das Rohmanusript, wobei die Hauptgedanken und vor allem der Duktus und die Ziele einvernehmlich festgelegt werden. Dazu kommt noch, daß die Visualisierung ganz genau vorbereitet wird. Dann wird das eigentliche Sendemanuskript erstellt und in einer dritten kurzen Sitzung noch einmal verbessert.

Der Ablauf der Sendungen hat folgendes Grundschema:

1. Signation: Gleichbleibendes akustisches und visuelles Signal, anschließend wird das Thema der Sendung im Schriftsatz auf ein konkretes Bild aufgelegt.

2. Der Sprecher tritt auf, artikuliert die Fragestellung und das Thema.

3. Mindestens einmal wird der Sprecher ausgeblendet und zur Verdeutlichung werden Bilder, Szenen, Zeugen zu Wort und Bild gebracht oder eine Sequenz bildlich aufbereitet.

4. Gegen Schluß lädt der Sprecher zum Schreiben ein und spricht seine Adresse, die gleichzeitig auch im Schriftsatz sichtbar wird.

Im September 1975 übernahm Dr. Anton Fellner die inzwischen zur „Stabsstelle: Kirche im Fernsehen" aufgerückte Abteilung. Der Intendant des 1. Kanals, Gerhard Weis, übernahm die Sendung aus dem 2. Kanal, gab ihr am Samstag spätabends eine ideale Zeit und einige ganz energische Impulse.

\section{Das Publikum}

Am Ende der Sendung wird, wie gesagt, der Name und die Adresse des Sprechers eingeblendet und dazu noch zum Schreiben eingeladen. Die Sendung trifft nun in einer ganz bestimmten Zeit einen ziemlich scharf umrissenen Zuschauerkreis in einer ganz speziellen Empfangssituation. Niemand, weder vom ORF, noch vom Team konnte ahnen, was daraus wurde. Leider gibt es über die Sendung noch keinen Infratest ${ }^{1}$ oder eine Untersuchung, die geeignete Grundlagen liefern würden. Aber schon liegen bei jedem der Sprecher hunderte von Briefen und persönliche Kontakte vor, so daß sich die Schwerpunkte genau lokalisieren lassen.

Die Sendung ist, wie gesagt, am Samstag abend nach $10 \mathrm{Uhr}$, eingeklemmt zwischen einer langen Sportsendung und dem zum Begriff gewordenen „Samstagnachtkrimi”. Das zwingt den Sprecher, sofort voll da zu sein, sonst schaltet der Zuschauer ab und tut etwas anderes. Die Frage muß also unmittelbar berühren. Die Art und Weise der Beantwortung müssen überzeugen, die menschliche Behandlung muß anrühren.

Die Zielgruppe könnte man aus der gesammelten Erfahrung der letzten 3 Jahre so umschreiben:

$\mathrm{Zu}$ dieser Zeit und bei diesem Sendungsumfeld (Sport und Krimi) sind wohl ganz besondere Gruppen am Fernsehapparat. Sicher sind es nicht vorwiegend die sogenannten braven Kirchgänger, sondern eher der Kirche gegenüber abwartende, kritische, ja ablehnende Menschen. Besonders angesp rochen aber fühlen sich Menschen in Zweifel, in Not, suchende, fragende, zweifelnde Menschen. Wir leben heute in einer gnadenlosen Zeit. Die Menschen sind innerlich unruhig, unsicher, kontaktarm, hoffnungslos. Ich glaube, man kann sich überhaupt keine Vorstellung machen, was heute in jungen Menschen, in Ehen, in einsamen, kranken, gestrauchelten Menschen vor sich geht. Unsere Zeit, unsere Welt ist herzlos geworden. 
Die Kirche trifft diese Menschen nicht mehr, sie erreicht sie zum größten Teil nicht mehr. Dafür gibt es viele und bekannte Gründe. Die Gemeindeseelsorge bricht zusammen. Die Sprache und Botschaft der Bibel wird nicht mehr verstanden. Ein Vergleich drängt sich auf, und man kann leicht abschätzen, was er bedeutet. Der „Fernsehpriester" hat in diesen 5 Minuten mindestens gleich viel Menschen vor sich, wie sämtliche Priester Österreichs in den Sonntagsmessen. Und er spricht sie ganz persönlich an, sie hören ihm privat zu, und er hat vor allem solche Zuhörer, die alle anderen Priester am Sonntag nicht erreichen! Und das ist eine ungeheure Chance, zugleich aber eine schwere, unerhört schwere Aufgabe.

Die Empfangssituation: Niemand von den Beteiligten hat je geahnt, welche Folgen diese Sendung haben würde! Da erscheint plötzlich auf dem Fernsehschirm ein sympathischer Mensch, ein Priester, der ganz menschlich spricht. Der sich anbietet, zu raten, zu helfen. Aus der Wirkungsforschung der Medien wissen wir, daß einer der Hauptfaktoren der Wirkung der sogenannte Heimeffekt ist. Und der besagt, daß der Zuschauer in seinem Intimbereich, in seinem Privatzimmer einen Gast empfängt, der mit ihm redet. Der wird sozusagen zum ,illusionären Partner”. Aus Untersuchungen der Action-serien wissen wir ja, daß die Helden nicht verheiratet sind! Sie werden dadurch leichter zu Identifikationsfiguren. Ein Beispiel: Professor Zöchbauer berichtet, ein Nachrichtensprecher (!) habe von einer alten Dame 1.000.- S erhalten mit dem Satz: Zum Dank dafür, daß Sie mich gestern abend in den Nachrichten so nett angeschaut haben! Und genau dieser human touch macht es möglich, muß offensichtlich vorhanden sein, damit die grausame Kontaktlosigkeit, die furchtbare Einsamkeit, die Mauer der Abgeschlossenheit durchbrochen werden kann, Wieder ein Beispiel: Eine 20jährige Frau ruft mich an, sie möchte mit mir reden. Sie fährt weit mit dem Zug, sie geht eine halbe Stunde zu Fuß, um zu mir zu kommen, und dann erzählt sie: Sie sei Sekretärin in einem Großbetrieb und erfülle ihren Beruf bestens. Aber nach Arbeitsende gehe sie nach Hause, schließe sich ein und habe Angst, mit jemand über irgend etwas zu reden. Auf meine Frage: Aber warum reden Sie denn jetzt so normal mit mir?, antwortet sie: Ja mit Ihnen ist es etwas anderes. Mit Ihnen habe ich ja schon im Fernsehen gesprochen.

\section{Die Bilanz}

Die Reaktion: Im ersten Jahr war die Reaktion nicht überwältigend, aber doch so ermutigend, daß wir die Sendung fortführten. Heute, nach 3 Jahren, sieht die Situation so aus: Ich schreibe in diesem Jahr über 1000 Briefe, habe in der Woche mindestens 3 Besuche, Besuche von Menschen, die oft $100 \mathrm{~km}$ weit fahren. Einer der Sprecher, P. Dr. Berthold Mayr, hat auf eine Sendung über 300 Briefe bekommen! Der Briefund Besucherstrom ist am stärksten während der 6 Wochen der Sendung, aber er reißt heute überhaupt nicht mehr ab.

Die Schreiber: Interessant ist in diesem Zusammenhang folgendes: Die meisten Briefe beginnen so: "Ich habe Sie im Fernsehen gesehen, zu Ihnen habe ich Vertrauen, und darum möchte ich Ihnen mein Anliegen schildern". Und dann kommt ein ganz anderes Thema, als es in der Fernsehsendung behandelt wurde. Das heißt, wichtig ist in erster Linie nicht das Thema, sondern die menschliche Art, wie der Sprecher über irgend eine Frage spricht. Damit signalisiert der Sprecher, daß er vertrauenswürdig ist, daß er auch als Priester ganz Mensch ist, daß er Hilfe verspricht, daß er Hoffnung gibt. Weiter ist sehr interessant, daß es kaum Schimpfbriefe gibt. Wenn jemand mit dem 
Thema oder der Art nicht einverstanden ist und Mißverständnisse auftreten, dann ist es in den allermeisten Fällen der Anlaß zu einem weiteren Gespräch. Die Schreiber kommen aus allen Bevölkerungsschichten, Altersstufen und Themenbereichen. Sie umfassen vor allem die großen Sorgenbereiche Erziehung, Ehe, Nöte, Katastrophen. Aber gleich an zweiter Stelle stehen tiefreligiöse Probleme, Sinnfragen, Auseinandersetzung mit der Amtskirche, Fragen nach dem Sinn des Leidens und den letzten Dingen.

Die Hilfe: Kann man durch eine solche Sendung überhaupt helfen? Wirksam helfen? Es gibt dazu sehr ernst zu nehmende kritische Stellungnahmen. Der Kontakt läuft über 3 Kanäle. Briefe, Telefon, Besuche². Das am meisten benutzte Medium ist der Brief, und hier lautet die Frage: Ein Brief ist doch keine Hilfe? Das ist ja nur ein Trost. Und überhaupt, wenn man einen Brief schreibt, dann dauert es mindestens einige Tage, und inzwischen ist der Absender bereits in einer anderen Situation. Wenn man aber so viele Briefe schreiben muß, dann muß es doch schnell gehen. Hat man überhaupt Zeit und Möglichkeit, den Schreiber wirklich zu verstehen, effektiv zu helfen? Diese Bedenken sind durchaus zutreffend. Dessen ist sich jeder Sprecher leider nur zu gut bewußt. Aber man kann mit einem Brief auch sehr viel tun. Allein schon eine Antwort, ein persönliches Schreiben bedeutet viel: Ich habe jemand, der mich ernst nimmt. Der mich für so viel wert hält, daß er mir persönlich schreibt. Hier spricht kein Amt (darum sind wir gegen jede Institutionalisierung mit Apparat). Dieser ganz persönliche Kontakt ist sicher das Entscheidende und das Wertvolle. Natürlich kann man die meisten Probleme nicht lösen. Aber ist lösen möglich und überhaupt gewollt? Wir wollen ja nicht Entscheidungen für andere fällen, sondern wir wollen helfen, Entscheidungen vorzubereiten, die der einzelne selbst trifft. Ein Brief ist immer eine schwienge Sache. Trifft man überhaupt das Problem? Trifft man mit der Art und Weise des Briefes den Schreiber? Sagt man genau, was er verträgt, nicht zu viel, nicht zu wenig, nichts Falsches? Ein Brief ist aber immer ein Beginn, um aus einem Konflikt einen Prozess zu machen. Ist nicht schon alles getan, was möglich ist, wenn man dem Schreiber sagt: Du hast ein schweres Problem, nimm es an, kämpfe Dich durch, Du wirst sehen, es wird gehen, und es wird gut.

Viele Menschen sind aber mit einem Brief nicht zufrieden. Es entstehen richtige Briefketten, oder es entstehen Telefongespräche, oder der Schreiber will unbedingt das persönliche Gespräch. Und hier sind die Schwierigkeiten überaus groß. Nicht nur die Entfernung, die Terminnot und andere Hindernisse sind schwer zu überwinden. Und meist ist es mit einem Gespräch noch nicht getan. Denn in vielen Fällen geht es um sehr schwierige, langwienige Lebenseinstellungen und Leben sänderungen. Sicher aber ist dieser erste Kontakt, was immer er bringt, dieser erste Anstoß, nicht nur entscheidend, sondern notwendig.

\section{Der Ausblick}

Es gibt eine Telefonseelsorge, es gibt aber auch eine Fernsehseelsorge! Und sicher kann man eines sagen: Wir haben bisher manche und gute, weil wirkungsvolle Verkündigungssendungen im Fernsehen gemacht. Dies aber ist die erste Seelsorgesendung. Niemand konnte ahnen, daß sie eine solche Wirkung haben würde. Heute erfüllt uns das mit Freude, weil wir helfen können, aber auch mit großer Sorge, weil wir es nur schwer bewältigen können.

Diese Sendung ist eine ungeheuere Chance. Niemand will anderen Versuchen und Möglichkeiten der Seelsorge ihre Bedeutung und Berechtigung absprechen. Aber man 
stelle sich einmal vor: Jede Sendung hat einen garantierten Zuhörerkreis zwischen 500.000 und 1.000.000 Zuhörer. Dabei sind ganz Österreich und alle Anrainerstaaten eine einzige Seelsorgegemeinde. Diese Sendung erfaßt aber einen Kreis, der an den Zäunen der Straßen steht. Es sind die Hilflosen, die Verzweifelten, die Suchenden. Es sind Menschen, die Jesus einlädt: Kommt alle zu mir, die ihr mühselig und beladen seid, ich will euch erquicken. Es ist keine spektakuläre Sendung. Es sind die Stillen, die Armen, die Kranken. Ihnen gerade aber gehörte die besondere Sorge und Mühe Jesu. Und darum sind wir uns voll bewußt, diese Sendung darf nicht aufhören. Diese Sendung ist eine ungeheure Chance für die Kirche.

Diese Sendung aber übersteigt die Möglichkeiten der Sprecher. Die einzelnen Priester sind überfordert. Rein arbeitsmäßig, dabei stehen die Sprecher im Dilemma. Sie müssen mitten in der Seelsorge stehen, damit sie ganz überzeugend wirken, und dann haben sie so viel Arbeit, daß man diese Sendung nebenbei nicht mehr bewältigen kann, und deshalb kann man nur sagen: Entweder der Sprecher bekommt Entlastung und Hilfe, oder er muß die Konsequenzen ziehen und ausscheiden. Doch Hilfe scheint möglich. Zunächst durch einen Arbeitskreis von Fachleuten. Der Sprecher ist oft nur die Vermittlungsstelle. Er braucht den Rechtsanwalt, er braucht den Arzt, er braucht den Suchtgiftexperten, die Caritas, die Eheberatung, den Sozialarbeiter. Und er braucht einfach Hilfe zum Briefeschreiben, die Sekretärin, den Pfarrhelfer.

Ganz von selbst ist diese Sendung in die jetzige Gestalt und Wirkung hineingewachsen. Würde sie aufhören, würde eine ganze Gruppe von Menschen die Hoffnung auf Hilfe verlieren. Aber auch die Kirche würde ihr ganz menschliches Gesicht verlieren und würde sich dadurch einer ungeheuren Chance begeben.

\section{Anmerkungen:}

1 Der ORF ist durch sein Statut verpflichtet, regelmäßig Untersuchungen über das Programm machen zu lassen und zwar von Firmen, die vom ORF unabhängig sind, damitein e objektive Beurteilung gewährleistet ist.

2 Hier wären 2 Fragen unbedingt weiter zu verfolgen:

1. Warum muß meistens ein Medium, Brief oder Telefon dazwischengeschoben werden? Offensichtlich ist es für viele Menschen schwer, einen direkten Kontakt aufzubauen. Deshalb das anonyme Papier und das unpersönliche Telefon. Brief und Telefon erlauben, daß man sich vollständig frei aussprechen kann, ohne seine Anonymität vollends abzulegen.

2. Was ist das, ein Brief, und welche Funktion hat ein Brief? Offensichtlich ist ein Brief ein Ventil. Man kann sich etwas von der Seele schreiben (Tagebuch), und so wird dem Schreiber vieles klarer. Der Brief hat aber einen konkreten Adressaten. Das heißt: Im Hintergrund steht ein Mensch, der versprochen hat, sich zu kümmern, sich anzunehmen, der einen mag, der einen also zu verstehen versucht. 


\section{SUMMARY}

The "Chruch and TV" Department of th Austrian Radio and Television Network (ORF) has for about three years aired a programme entitled "Questions the Christian asks." This programme aims at pastoral contacts and care rather than evangelisation. The five minute transmission is on Saturday evenings after 10 o'clock, sandwiched between Sport and a Detective programme. The content is determined by viewers themselves, and pictures and illustrations are used with the subjects treated. Its success seems to be considerable, and there are 1,000 personal letters a year, many phone calls, and also visits. The programme fills a gap in the unsuspected loneliness of modern man and his deep human sorrows which cannot be formulated or analysed when alone. The "home effects" of television, meeting man in his private and personal environment, helps to build up a semblance of partnership. The positive attitude of the programme and a certain human warmth of the speakers manages to break through the block built up against human relationships. Letter and phone are media which are on the one hand sufficiently anonymous to let the loneliness remain, but, on the other hand, can build up a personal relationship. This programme demonstrates a new form of pastoral care which could provide openings for the Church. The author is one of the speakers in the series and at the same time parish priest in a small Tyrolean village.

\section{RÉSUMÉ}

La section "Kirche im Fernsehen" de la ORF (Radio Autrichienne) a, depuis trois ans, une émission pas tant transmise comme proclamation, mais tout entière dirigée vers les contacts et l'aide pastoraux: "Fragen der Christen". Elle dure cinq minutes et est fixée le samedi soir après 10 heures, encastrée entre une grande émission sportive et un film policier. Termes du problème et contenu sont un problème posé par le public. La préparation se passe également autant que possible en images et en scènes. L'effet, dont on ne se doutait pas, est large et profond (plus de 1000 lettres par an, beaucoup d'appels telephoniques et de visites). Vraisemblablement cette émission rencontre une immense solitude et les misères humaines les plus lourdes qui ne peuvent plus être articulées et travaillées. La télévision a, par l'intermédiaire du soi-disant "home-effect", la possibilité de pénétrer dans la sphère intime de l'homme et de construire de façon toute personnelle une soi-disant participation illusoire. La façon positive de traiter un thème et la chaleur humaine du speaker franchit le mur du son des barrières de contact. Lettre et téléphone sont des moyens qui d'une part sont assez anonymes pour que l'homme sorte de sa solitude, mais qui d'autre part construisent une rencontre toute personnelle. L'émission est une forme de pastorale toute nouvelle et offre d'immenses possibilités.

\section{RESUMEN}

La sección "Iglesia en la televisión" de la ORF (televisión austriaca) emite desde hace tres años el programa "Preguntas de cristianos", orientado no tanto a la proclamación de la palabra como al contacto y la ayuda personal. Dura 5 minutos y es emitido los sábados después de las diez de la noche, empanado entre una gran emisión deportiva y una película politiciaca. El contenido viene determinado por un problema planteado por el público. La elaboración incluye también, en lo posible, fotografias y escenas. La resonancia del programa es inesperadamente amplia (más de 1.000 cartas al año, muchas llamadas telefónicas y visitas). Al parecer esta emisión se abre paso hasta estados de inmensa soledad y de grave necesidad humana. Mediante el llamado "Home effect" la televisión tiene la posibilidad de introducirse en la esfera intima del hombre y de crear una especie de amistad ilusoria muy personal. El modo personal de tratar los temas y el calor humano que irradia el locutor atraviesan los muros de la incomunicación. Las cartas y las llamadas telefónicas son medios, por otra parte lo suficientemente anónimos, para que el hombre salga de su soledad y entable un contacto totalmente personal. La emisión es un modo totalmente nuevo de pastoral y ofrece posibilidades increibles. 